Methods The Georgians Organized Against Lupus (GOAL) is a cohort of validated patients with SLE living in Atlanta, predominantly derived from the population-based and Centers for Disease Control and Prevention (CDC) funded Georgia Lupus Registry. Participants have been surveyed annually, including sociodemographics, health insurance, disease activity (Systemic Lupus Activity Questionnaire), and damage (Self-Administered Brief Index of Lupus Damage). Self-reported health insurance was categorized into no insurance, private, Medicare, Medicaid, and Medicare/Medicaid. Those reported being in a different category the year before were classified as having changed insurance.

Results An average of 642 individuals were surveyed annually from 2012 to 2016. At baseline, the average age was 46.4 \pm 13.4 and disease duration was $13.6 \pm 9.2$ years. $93.6 \%$ were female and $78.5 \%$ black. $35.1 \%$ had a high school educational level or less, $45.8 \%$ were at or below the Federal poverty level, $34.6 \%$ were married or with a partner, and $35 \%$ were employed. Figure 1 shows the distribution of insurance categories from 2012 through 2016. Compared to the year before, $23.8 \%$ changed insurance in $2013,22.2 \%$ in $2014,24.1 \%$ in 2015 , and $26.8 \%$ in 2016. Those who changed insurance tended to be black, lower in educational attainment, poorer, unemployed, and have greater disease activity and damage.

Conclusions In a population-based cohort in Georgia, the majority with SLE have private insurance and Medicare, which has grown over time while those uninsured have dropped. This is in line, but greater in magnitude, with the decrease in uninsured seen with the Affordable Care Act. Georgia is one of the states that has not expanded Medicaid resulting in flat enrollment. There also appears to be an increase in those who switch insurance categories. In this era of fluctuating health care policy, it is important to learn how types of and changes in coverage affect health care utilization, disease treatment and outcomes, self-reported health, and mortality in SLE, particularly given the disproportionate impact on vulnerable groups. Studies utilizing administrative data should also be aware of medical coverage distributions and regional variations in policy that impact these distributions.

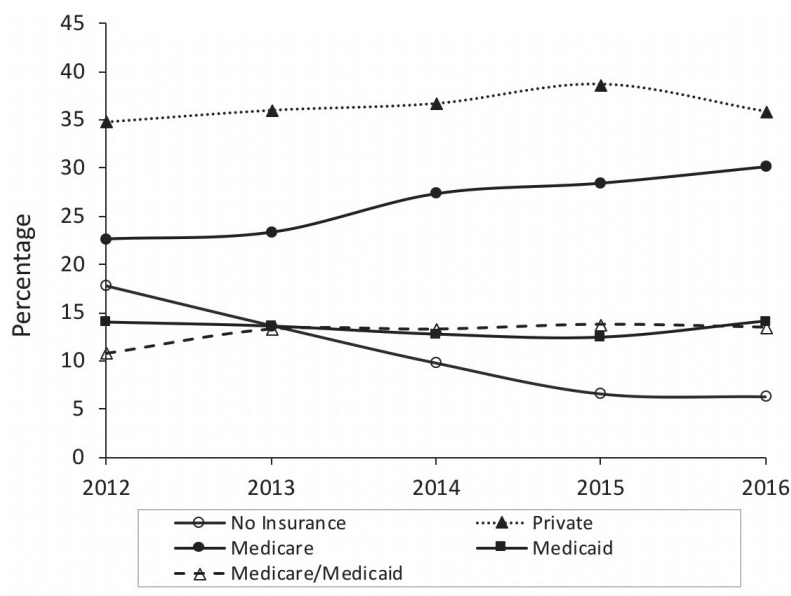

Abstract BD-10 Figure 1 Longitudinal distribution of self-reported medical coverage

Acknowledgements Supported by 3U58DP001487-05 W2 and U01DP005119 (CDC).

\section{BD-11 LACK OF HETEROGENEITY ACROSS RACE/ETHNIC GROUPS IN THE RISK OF COMMON COMORBIDITIES AMONG PATIENTS WITH SLE COMPARED TO THE GENERAL POPULATION IN A LARGE HEALTHCARE SYSTEM IN CALIFORNIA}

${ }^{1}$ Titilola Falasinnu*, ${ }^{2}$ Jiang Li, ${ }^{2}$ Sukyung Chung, ${ }^{3}$ Latha Palaniappan, ${ }^{1,4}$ Julia F Simard. ${ }^{1}$ Department of Health Research and Policy, Stanford School of Medicine, Stanford, California, USA; ${ }^{2}$ Palo Alto Medical Foundation Research Institute, Palo Alto, California, USA; ${ }^{3}$ Division of Primary Care and Population Health, Stanford School of Medicine, Stanford, California, USA; ${ }^{4}$ Division of Immunology and Rheumatology, Stanford School of Medicine, Stanford, California, USA

\subsection{6/lupus-2018-Ism.35}

Background Systemic lupus erythematosus (SLE) disproportionately affects racial/ethnic minorities and is an independent risk factor for pneumonia, infections, renal disease and cardiovascular diseases (CVD). There are also racial/ethnic differences in these common comorbidities. There is limited understanding of how much inequality or heterogeneity SLE confers independently of the underlying disease pathways of comorbidities between race/ethnic groups. We examined the patterns, strength and direction of the risk of comorbidities in race/ethnic groups with SLE compared to controls. We hypothesized that those with SLE will have increased risk of comorbidities and that these associations will be strongest for racial minorities and weakest for non-Hispanic whites (NHW).

Methods We defined a cohort using electronic health records data (2005-2016) from a large healthcare organization in California serving patients of diverse racial/ethnic backgrounds. The eligible population included SLE patients and age-sexrace/ethnicity matched non-SLE patients ( $\geq 18$ years of age). SLE diagnosis was the primary exposure defined using ICD9: 710.0 and ICD10: M32.1, M32.8, and M32.9. The following outcomes were identified: pneumonia, infections, renal disease and CVD. For each racial/ethnic group, we calculated the proportion of incident comorbidities among SLE cases and controls, and then built adjusted logistic regression models for each outcome with SLE as the exposure.

Results We identified 1,290 SLE cases and 12900 controls. The median age at baseline was 43 years and $92 \%$ of the cohort was female. Among SLE cases, 38\% were NHW, 13\% were Hispanic, $4 \%$ were Black, and $25 \%$ were Asian/Pacific Islander. Compared to controls, SLE cases had higher incidence of pneumonia (32\% vs 18\%), infections (46\% vs 26\%), renal disease $(16 \%$ vs $3 \%)$ and CVD $(45 \%$ vs $22 \%)$. The association persisted with odds ratio (OR) of 1.5 to 3 , after adjusting for confounders $(p<0.01)$. In subgroups analysis, ORs were similar across all racial/ethnic groups. We found no evidence for heterogeneity in the patterning of increased odds of the comorbidities between race/ethnic groups. For example, NHW, Blacks, Hispanics and Asians with SLE had between 23 times increased odds of CVD compared to controls.

Conclusions Our findings suggest that SLE similarly increases the risk of pneumonia, infections, renal disease and cardiovascular diseases (CVD) across racial/ethnic groups. Understanding the reasons for the lack of heterogeneity is important for targeting preventative interventions among patients with SLE.

Acknowledgements The authors would like to gratefully acknowledge the National Heart, Lung, and Blood Institute for funding this research. 


\section{Clinical Sciences}

\section{CS-01 DIFFERENTIAL EXPRESSION OF THE FSTL-1 PROTEIN AMONG PATIENTS WITH SYSTEMIC LUPUS ERYTHEMATOSUS}

\begin{abstract}
${ }^{1,2}$ Adriana Carlomagno* ${ }^{*}{ }^{3}$ Fiorella Cavalleri, ${ }^{1,4}$ Paola Contreras, ${ }^{3}$ José Badano, ${ }^{2}$ Álvaro Danza, ${ }^{5}$ José Mario Sabio, ${ }^{1}$ Carlos Escande. 'Laboratory of Metabolic Diseases and Aging - Institut Pasteur de Montevideo. Montevideo - Uruguay; ${ }^{2}$ Clinical Medical '2' Faculty of Medicine - Hospital Pasteur - Montevideo - Uruguay; ${ }^{3}$ Laboratory of Human Molecular Genetics - Institut Pasteur de Montevideo. Montevideo - Uruguay; ${ }^{4}$ Department of Physiology, Faculty of Medicine - Montevideo - Uruguay; ${ }^{5}$ Unit of Autoimmune diseases, Virgen de las Nieves Hospital - Granada - Spain
\end{abstract}

\subsection{6/lupus-2018-Ism.36}

Background There is an increasing prevalence of insulin resistance and metabolic syndrome in Systemic Lupus Erythematosus (SLE). Follistatin-like 1 (FSTL-1) is a secreted glycoprotein recently identified as a proinflammatory cytokine and emerging as a potential mediator of inflammation. Its plasma levels are elevated in rheumatoid arthritis and correlate with disease activity. In fact, FSTL-1 has been proposed as a marker of autoimmune diseases. However, it is not clear whether this protein plays a role in SLE. We aimed to evaluate the plasma FSTL-1 value of patients with SLE and the interactions between their plasma concentration, metabolic syndrome variables, markers of activity and disease damage.

Methods Plasma concentration of FSTL-1 of 40 female SLE patients and 20 healthy controls was measured by enzymelinked immunosorbent assay (ELISA). Metabolic variables, activity and disease damage were measured and analyzed. The activity of SLE was determined by Systemic Lupus Erythematosus Disease Activity Index (SLEDAI-2K). Damage accumulated was calculated by the Index of Damage SLICC/ACR (SDI Damage index). A one-way ANOVA test was performed to assess the difference between the mean FSTL-1 values in different groups. Interactions between variables of the database and their effect on FSTL-1 was evaluated using a regression tree technique.

Results Healthy controls and SLE females attended on an outpatient basis had a mean of $38 \pm 16$ and $40 \pm 13$ years old respectively. SLE patients had low levels of activity and damage measured both by the scores and by the individual parameters of inflammatory activity. The mean value of FSTL-1 was $12213 \pm 1424 \mathrm{pg} / \mathrm{ml}$ for healthy individuals. Of the SLE patients $57 \%$ had a mean value of $4855 \pm 662.8 \mathrm{pg} / \mathrm{ml}$ and $43 \%$ had a mean of $68000 \pm 4789 \mathrm{pg} / \mathrm{ml}$. There was a significant difference between the subgroup of SLE with high value of FSTL-1 compared to the control (p-value $<0.001)$ and the subgroup with

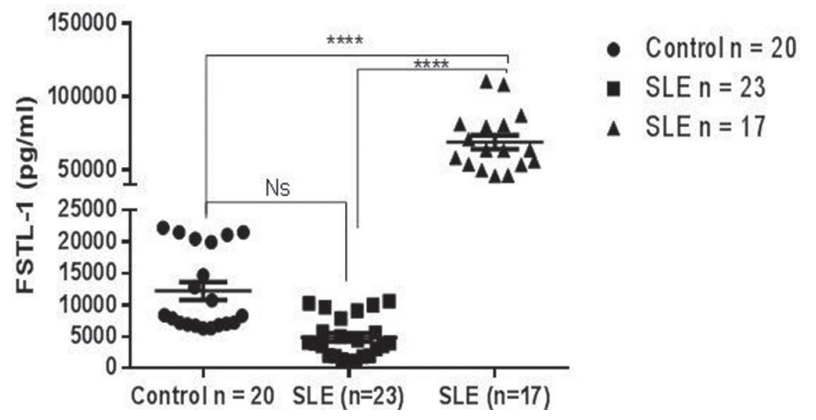

low value of FSTL-1 (p-value<0.001) (figure 1). From the regression analysis, we found a correlation between increasing plasma concentrations of FSTL-1 and presence of variables of metabolic syndrome (insulin, cholesterol and leptin) in patients with SLE. No correlation was found between FSLT-1 value and the activity or damage of the disease.

Conclusions FSTL-1 is significantly elevated in patients with SLE compared to controls. Moreover, elevated levels were observed in SLE patients with elevated leptin, cholesterol or insulin. More studies are needed to determine if there is a pathogenic role, and if this population have a worse longterm cardiovascular prognosis.

\section{CS-02 THE LUPUS COHORT IN THE NEW CARRA REGISTRY: THE FIRST YEAR OF ENROLLMENT}

${ }^{1}$ Aimee 0 Hersh*, ${ }^{2}$ Mary Beth Son, ${ }^{3}$ Stacy Ardoin, ${ }^{4}$ Deborah Levy. ${ }^{1}$ University of Utah, Salt Lake City, UT, USA; ${ }^{2}$ Boston Children's Hospital, Boston, MA, USA; ${ }^{3}$ Nationwide Children's Hospital, Columbus, OH, USA; ${ }^{4}$ Hospital for Sick Children and University of Toronto, Toronto, Canada

\subsection{6/lupus-2018-Ism.37}

Background The Childhood Arthritis and Rheumatology Research Alliance (CARRA) aims to collect meaningful clinical data on all children affected by rheumatic disease in North America as a basis for performing high quality clinical and translational research. The new CARRA Registry (building on a prior CARRA registry with a minimal dataset) has enrolled pediatric patients with systemic lupus erythematosus (SLE) and related conditions since March 2017. We sought to describe the popula-

Abstract CS-02 Table 1 Components of SLEDAI Present within 30 days of Baseline Visit $(n=184)$

\begin{tabular}{ll}
\hline SLEDAl Component & $\mathrm{N}(\%)$ \\
\hline Seizure & $1(0.5 \%)$ \\
Psychosis & $2(1 \%)$ \\
Organic Brain Syndrome & $1(0.5 \%)$ \\
Visual Disturbance & $1(0.5 \%)$ \\
Cranial Nerve Disorder & $1(0.5 \%)$ \\
Lupus Headache & $8(4 \%)$ \\
Cerebrovascular Accident & 0 \\
Vasculitis & $6(3 \%)$ \\
Arthritis & $24(13 \%)$ \\
Myositis & $8(4 \%)$ \\
Urinary Casts & $25(14 \%)$ \\
Hematuria & $13(7 \%)$ \\
Proteinuria & $13(7 \%)$ \\
Pyuria & $10(5 \%)$ \\
Rash & $32(17 \%)$ \\
Alopecia & $13(7 \%)$ \\
Mucosal Ulcers & $19(10 \%)$ \\
Pleurisy & 0 \\
Pericarditis & 0 \\
Low Complement & $72(39 \%)$ \\
Fevereased DNA Binding & $50(27 \%)$ \\
Leukopenia & $14(8 \%)$ \\
\hline & $8(4 \%)$ \\
\hline & $22(12 \%)$ \\
\hline &
\end{tabular}

Abstract CS-01 Figure 1 